\title{
Effect of strengthening lower trapezius muscle on scapular tipping in patients with diabetic frozen shoulder: A randomized controlled study.
}

\author{
Huda B. Abd Elhamed ${ }^{1}$, Ghada M. Koura1, Hamada Ahmed Hamada ${ }^{2 *}$, Yassmin E. Mohamed ${ }^{2}$, Rami \\ Abbas $^{3}$ \\ ${ }^{1}$ Musculoskeletal Disorders and its Surgery Faculty of Physical Therapy, Cairo University \\ ${ }^{2}$ Lecture of Biomechanics, Faculty of Physical Therapy, Cairo University \\ ${ }^{3}$ Department of Physical Therapy, Faculty of Health Sciences, Beirut Arab University, Beirut, Lebanon
}

\begin{abstract}
Background: The muscle imbalances between upper and lower trapezius muscle activity are related to abnormal scapular motion in patients with frozen shoulder. This explains the importance of rehabilitation of the lower trapezius muscle in addition to common stretching and mobilizing treatment protocols for patients with frozen shoulder.

Purpose: This study aims to investigate the effect of additional strengthening exercises of lower fibers of trapezius muscle on the scapular tipping in patients with Diabetic Frozen Shoulder (DFS).

Subjects and methods: Thirty patients with DFS were randomly assigned into two equal groups $A$ and B. Both groups received a traditional physical therapy program, however group B additionally received strengthening exercises for lower fibers of trapezius. Baseline and post-treatment assessment for the scapular tipping were evaluated by using (A-T) distance test.

Results: After treatment; mixed design MANOVA revealed significant improvement in scapular tipping (A-T) distance from supine position, supine position with scapula retraction, standing position, and standing position with scapula retraction, for group $B$ more than group $A(P<0.05)$. There were significant improvements in scapular tipping when comparing the post-treatment mean value with pretreatment in group $B$ only $(\mathbf{P}<\mathbf{0 . 0 5})$.

Conclusion: Strengthening of the lower trapezius fibers plus traditional physical therapy program yields improvement in scapular tipping in patients with DFS more than traditional physical therapy alone.
\end{abstract}

Keywords: Diabetic frozen shoulder, Scapular tipping, Strengthening lower fibers of trapezius.

Accepted on November 2, 2017

\section{Introduction}

Frozen shoulder syndrome, also known as adhesive capsulitis [1], is a clinical entity that refers to a stiff and painful shoulder causing major functional impairment $[2,3]$. These functional impairments limit everyday activities, especially ones which comprise overhead reaching activities. These impairments might range for a period of several months to several years, in addition to shoulder pain disturbing sleep at night on the affected side [4]. It has been estimated that this syndrome affects up to $5 \%$ of the general population [5], with peaked incidence rates occurring between the fifth and sixth decades. It affects women more than men, and is mostly associated with diabetes mellitus as a systemic condition [5]. Frozen shoulderrelated dysfunction can affect individuals' function independently and consequently decrease the quality of life. Frozen shoulder may be either primary idiopathic or secondary to a systemic disease, such as diabetes mellitus and thyroid disorders $[2,6]$.
There are four clinical stages of adhesive capsulitis: in stage I, lasting up to 3 months, patients suffer achy pain at rest and sharp pain at end ranges of motion with sleep disturbances; in stage II, referred to as the "painful" or "freezing" stage, lasts from 3 to 9 months in which pain causes substantial gradual loss of motion in all directions; in stage III, known as the "frozen" stage, includes pain and loss of motion from 9 to 15 months [7]; and in stage IV, known as the "thawing" stage, ranging from 15 to 24 months where pain resolves gradually but with persistence of stiffness [8].

In the past two decades, many research studies focused on assessing the role of the scapula in upper limb function. It had been shown that when scapular stabilizers weaken and/or when alteration occurs in their kinematics, stress to the anterior capsular shoulder structures increases leading to rotator cuff compression and decreased neuromuscular performance of the shoulder [9]. Yet, studies scrutinizing the role of the scapula in shoulder instability and adhesive capsulitis are still scarce [10]. 
Diminished strength of the scapular and thoracic muscles may theoretically cause abnormal positioning of the scapula, in addition to instabilities in scapulohumeral rhythm, and generalized dysfunction of the shoulder complex [11]. Among the mostly affected muscles pertaining to abnormal shoulder movement are the serratus anterior and lower trapezius muscles which engulf the scapulothoracic joint [12].

Patients with adhesive capsulitis have significantly weaker lower trapezius muscles compared to asymptomatic controls. Additionally, higher Electromygraphic (EMG) ratios of upper trapezius to lower trapezius during arm elevation were found in patients with adhesive capsulitis when compared to asymptomatic subjects [13]. These muscle imbalances are accused to increase elevation and upward rotation of the scapula during elevation of the glenohumeral joint in both frontal and sagittal planes [14]. Consequently, in patients with adhesive capsulitis, the exercises that generally activate several scapulothoracic muscles, reducing activity of the upper trapezius and selectively stimulating the lower trapezius muscle may be advantageous [13]. The increases in the lower trapezius muscle activation leads to posterior tipping of the scapula. This correct mechanics avoids closer proximity between the anterior acromion and rotator cuff tendons during elevation of the arm [15]. Thus, explaining the importance of rehabilitation of the lower trapezius muscle in addition to common stretching and mobilizing treatment protocols for patients with frozen shoulder. Therefore, lower trapezius muscle strengthening exercises can be hypothesized to avoid abnormal kinematics and shoulder impingement in patients with adhesive capsulitis.

\section{Study Design}

The study was designed as a prospective, randomized, singleblind, pre-post-test, controlled trial (RCT).

\section{Study participants}

In total, 35 patients with diabetic frozen shoulder, referred to the out-clinic of the Faculty of physical therapy, Cairo University, were enrolled and assessed for their eligibility to participate in the study. Their age ranged from 40 to $60 \mathrm{y}$. Before randomization, five patients were excluded, two patients with secondary arthritis, one patient with fracture; two patients chose to withdraw their consent for participation in the study. Thirty patients with DFS were randomly assigned into two equal groups, by using a shuffled deck of cards (e.g., No. single-group A, No. double-group B) [16]. No subjects dropped out of the study after randomization. Written informed consent was obtained from all subjects before the baseline evaluation. Ethical approval was obtained from the institutional review board at Faculty of physical therapy, Cairo University before study commencement. The study followed the Guidelines of Declaration of Helsinki on conduction of human research [17].

The inclusion criteria were patients who had shoulder pain and restriction in ROM (50\% loss of passive ROM of the shoulder joint relative to the unaffected side in at least three directions, i.e. external rotation, abduction, internal rotation and flexion) for a duration of at least 3 months and in whom no treatment other than analgesics had been prescribed within the past 3 months and no radiographic abnormal findings on anteroposterior, axillary or scapular y-view shoulder radiographs were found [18]. Exclusion criteria were bilateral shoulder involvement, history of previous surgery on the shoulder, shoulder fracture, cancer, glenohumeral or acromioclavicular arthritis, inflammatory disorders, bleeding disorders, presence of severe osteoporosis, pulmonary diseases, any neuromuscular disorders, pregnancy, and unwillingness to participate in the study [19].

\section{Outcome measures}

The scapular tipping was measured before and after the treatment program using (A-T) distance test. The measurement of the distance between the posterior border of the acromion and the table was performed as described by Host, 1995 [20]. Such measurement was found to have intraclass correlation coefficients greater than .88 for the (A-T) distance test [21]. It was measured from four positions: supine lying, supine with shoulder retraction, standing, and standing with shoulder retraction. First position, the patient was supine and instructed to relax his shoulder. Such position has been claimed to reduce muscular activity is needed for scapular stability. The investigator measured the distance between the posterior border of the acromion and the table (measured vertically with a tape measure). Second position, the investigator repeated this procedure while the patient was asked to actively retract both shoulders and to move both shoulders toward the table surface.

Third position, the patient was standing and the therapist instructed the patient to put his or her feet and thorax against the wall and to stand relaxed. The therapist measured the distance between the posterior border of the acromion and the wall. Fourth position, the investigator repeated this procedure from standing with the patient asked to actively retract both shoulder and to actively move both shoulders toward the wall surface [22].

\section{Treatment procedures}

The patients were received 3 sessions/w for $4 \mathrm{w}$ [23]. Group A: 15 patients had received the traditional treatment in form of therapeutic ultrasound, mobilization and home program. Group B: 15 patients had received strengthening exercises to lower fibers of trapezius plus the previously mentioned traditional treatment. Therapeutic ultrasound was applied through slow circular movements while the patient was sitting. $1 \mathrm{MHz}$ continuous ultrasound was used at dose of $1.5 \mathrm{~W} / \mathrm{cm}^{2}$ for 10 min [24]; while mobilization was performed for 4-5 $\mathrm{min}, 10$ repetitions for 3 sets.

Glenohumeral distraction: Patient was supine, with the affected arm in the resting position. The investigator supported the patient's forearm between his trunk and elbow. Investigator's hand in the patient's axilla with his thumb just 
distal to the joint margin anteriorly and fingers posteriorly. The other hand supported the humerus from the lateral surface. Investigator pulled the humerus laterally [25].

Glenohumeral caudal glide: To increase the abduction ROM. Patient was supine, with the affected arm abducted to the end of its available range. External rotation of the humerus was added to the end-range position as the arm approaches and goes beyond $90^{\circ}$. The investigator stood facing the patient's feet and stabilized the patient's arm against his trunk with the farthest hand from the patient. Slight lateral motion of the investigator's trunk provided grade I distraction. The web space of the investigator's other hand was just distal to the acromion process on the proximal humerus. The investigator glided the humerus in an inferior direction [25].

Glenohumeral posterior glide: To increase the flexion and internal rotation ROM. Patient was positioned supine, with the arm in resting position. The investigator stood between the patient's trunk and arm, and supported the arm against his trunk. The distal humerus was grasped with the investigator lateral hand. This hand provided grade I distraction to the joint. The lateral border of the investigator's top hand was just distal to the anterior margin of the joint, with the fingers pointing superiorly. This hand gave the mobilizing force, while the investigator glided the humeral head posteriorly by moving the entire arm as investigator was bending his knees [23].

Glenohumeral anterior glide: To increase the extension and external rotation ROM. Patient was prone, with the arm in resting position over the edge of the treatment table, supported on investigator's thigh. The investigator stabilized the acromion with padding. Investigator stood facing the top of the table with the leg closer to the table in a forward stride position. The investigator supported the patient's arm against his thigh with his outside hand, the arm positioned on his thigh provides a grade I distraction. The ulnar border of the other hand just distal to the posterior angle of the acromion process, with the fingers pointing superiorly; this hand gave the mobilizing force. The investigator glided the humeral head in an anterior and slightly medial direction. Investigator's both knees were bent so the entire arm was allowed to move anteriorly [23]. Home program exercise: wand exercises are traditional ROM exercises [25]. It presents lower forms of tensile stress. The patient grasped the wand with both hands, and the normal extremity guided and controlled the motion. Each exercise was applied for 10 repetitions via 3 sets.

Shoulder flexion and return: The wand was grasped with the hands a shoulder-width apart. The wand was lifted forward and upward through the available range, with the elbows kept in extension if possible. Scapulohumeral motion was performed smoothly, as not to allow scapular elevation or trunk elevation movement [25].

Shoulder horizontal abduction: The wand was lifted to $90^{\circ}$ shoulder flexion, while the elbow was kept extended. The patient pushed and pulled the wand back and forth across the chest through the available range, in a way not to allow trunk rotation [25].
Shoulder internal and external rotation: The patient's arms were positioned at the sides, and the elbows flexed at $90^{\circ}$. Rotation of the arms was accomplished by moving the wand from side to side across the trunk while maintaining the elbows at the side [25].

\section{Lower trapezius strengthening exercises}

The modified prone cobra and prone row exercises are considered the most effective exercises for targeted strengthening of the lower trapezius muscle [26].

A-Modified prone cobra: The participant lay prone on a wide bench with the arms by the sides and fingers pointing towards the toes. The participant extended his trunk, to raise the chest approximately $10 \mathrm{~cm}$ off the bench. The palms turned out so that thumbs pointed up towards the ceiling, the participant pulled his shoulder blades back and down as if reaching his fingertips towards the feet. Manual resistance was applied concurrently on shoulder blades and in a cephalic direction into the palms, resisting the movement of reaching for the toes. The participant attempted to hold this against constant amount of resistance, for 3-4 s. This exercise was repeated 10 times [26].

B-prone V-raise exercise: The participants laid prone on a bench with the shoulders elevated overhead and at an abduction angle to $120^{\circ}$ in line with the lower trapezius muscle fibres and with the elbows extended. The investigator stood beside the participant, the participant was asked to lift off the affected arm from the bench to ear level with thumbs pointing up towards the ceiling. Manual resistance was applied in a downward direction, just above the elbow joints [27]. The participant was asked to hold this against constant amount of resistance, for 3-4 s. This was repeated 10 times [28].

\section{Statistical analysis}

All statistical measures were performed using the Statistical Package for Social science (SPSS) program version 20 for windows. Descriptive analyses showed that the data were normally distributed and not violates the parametric assumption for the all measured dependent variables (scapular tipping from supine position, supine with retraction, standing and standing with retraction). Additionally, testing for the homogeneity of covariance using Box's test revealed that there was no significant difference with $p$ values of $>0.05$. The box and whiskers plots of the tested variables were done to detect the outliers. Normality test of data using Shapiro-Wilk test was used, reflecting that the data was normally distributed for all dependent variables. All these findings allowed the researchers to conduct parametric analysis. So, $2 \times 2$ mixed design MANOVA was used to compare the tested variables of interest at different tested groups and measuring periods. The alpha level was set at 0.05 .

\section{Results}

As indicated by the independent $t$ test, there were no statistically significant differences $(\mathrm{P}>0.05)$ between subjects in both groups concerning age, weight, and height (Table 1). 
Statistical analysis using mixed design MANOVA analysed thirty patients assigned into two equal groups. It revealed that there were significance within subject effect $(\mathrm{F}=40.451$, $\mathrm{p}=0.0001)$ and treatment $\times$ time effect $(\mathrm{F}=33.25, \mathrm{p}=0.001)$. As well as, there was significance between subject effect $(\mathrm{F}=1.758, \mathrm{p}=0.016)$. Table 2 presents descriptive statistic (mean $\pm \mathrm{SD}$ ) and multiple pairwise comparison tests (Post hoc tests) of all investigated variables. In the same context regarding within subject effect, the multiple pairwise comparison tests revealed that there were significant decreases $(\mathrm{p}<0.05)$ in the $(\mathrm{A}-\mathrm{T})$ distance from supine position, supine position with scapula retraction, standing position, and standing position with scapula retraction in the post-treatment condition compared with the pre-treatment in group B and no significant difference $(p>0.05)$ in group A. Regarding between subject effects multiple pairwise comparisons revealed that there were no significant difference in the pre-treatment period between groups $\mathrm{A}$ and $\mathrm{B}$ in the $(\mathrm{A}-\mathrm{T})$ distance from all tested positions. ( $p>0.05)$, and in the pre-treatment period between groups $\mathrm{A}$ and $\mathrm{B}$ in the (A-T) distance from all tested positions. However, the (A-T) distance from all tested positions decreased significantly in group B compared with group A in post-treatment period $(\mathrm{p}<0.05)$.

Table 1. Demographic characteristics of patients in both groups.

\begin{tabular}{lllll}
\hline Characteristics & Group A $(\mathbf{n}=\mathbf{1 5})$ & Group B $(\mathbf{n}=\mathbf{1 5})$ & t-value & P-value \\
\hline Age $(\mathrm{y})$ & $26.06 \pm 3.39$ & $25.06 \pm 3.36$ & 0.777 & 0.444 \\
\hline Weight $(\mathrm{Kg})$ & $67.4 \pm 7.83$ & $69.2 \pm 4.66$ & -1.036 & 0.309 \\
\hline Height $(\mathrm{cm})$ & $167.26 \pm 8.69$ & $170.33 \pm 7.47$ & -0.765 & 0.451 \\
\hline
\end{tabular}

*Significant level is set at alpha level<0.05

Table 2. Descriptive statistics and multiple pairwise comparison tests (Post hoc tests) of the dependent variables for both groups.

\begin{tabular}{|c|c|c|c|c|}
\hline & Group A & & Group B & \\
\hline A-T distance from & Pre-treatment & Post-treatment & Pre-treatment & Post-treatment \\
\hline Supine position & $10.13 \pm 2.06$ & $10.05 \pm 1.94$ & $10.26 \pm 2.59$ & $6.96 \pm 1.51$ \\
\hline Supine with retraction & $8.1 \pm 2.08$ & $8 \pm 2.05$ & $8.16 \pm 1.57$ & $5.23 \pm 1.11$ \\
\hline Standing position & $12.73 \pm 1.6$ & $12.5 \pm 1.08$ & $12.7 \pm 2.06$ & $9.9 \pm 1.47$ \\
\hline Standing with retraction & $10.83 \pm 2.76$ & $10.73 \pm 2.49$ & $10.33 \pm 1.89$ & $8.03 \pm 1.5$ \\
\hline \multicolumn{5}{|c|}{ Within groups (Pre vs. Post) } \\
\hline p-value & Supine position & Supine with retraction & Standing position & \\
\hline Group A & 0.857 & 0.691 & 0.463 & 0.682 \\
\hline Group B & $0.0001^{*}$ & $0.0001^{*}$ & $0.0001^{*}$ & $0.0001^{*}$ \\
\hline \multicolumn{5}{|c|}{ Between groups (group A vs. group B) } \\
\hline p-value & Supine position & Supine with retraction & Standing position & Standing with retraction \\
\hline Pre-treatment & 0.877 & 0.922 & 0.961 & 0.568 \\
\hline Post-treatment & $0.0001^{*}$ & $0.0001^{*}$ & $0.0001^{*}$ & $0.0001^{*}$ \\
\hline
\end{tabular}

\section{Discussion}

In previous studies, scapular alterations have been assessed in patients with frozen shoulder, but treatment programs were mainly focusing on pain relief and improvement in ROM. As reported by Lin et al. [13], increased upper trapezius activity and imbalance of upper trapezius/lower trapezius activity have been reported in patients with adhesive capsulitis. Increased upper trapezius activity is clinically observed as a shrug maneuver, resulting in a type III (upper medial border prominence) dyskinesis pattern. This causes impingement due to lack of acromial elevation. Additionally, there is inhibition or delay in the activity of lower fibers of trapezius; this results in a type III/type II (entire medial border prominence) dyskinesis pattern, which causes impingement due to loss of acromial elevation and posterior tilting [29]. The insufficiency of the increased lower trapezius muscle activity should be an important consideration in the rehabilitation of frozen shoulder. Therefore, the aim of this study was to investigate the additional effect of strengthening exercises of lower fibers of trapezius muscle on the scapular tipping in patients with Diabetic Frozen Shoulder (DFS).

The results showed an obvious reduction in the scapular tipping ((A-T) distance) from all tested positions at the posttreatment period compared with pre-treatment for group $\mathrm{B}$ only. Additionally, after treatment there was significant improvement in scapular tipping (A-T) distance from supine position, supine position with scapula retraction, standing position, and standing position with scapula retraction) of group B than group A. 
These findings reflected that the improvement in scapular tipping in all patients immediately after the treatment might be caused by the additional effect of the strengthening exercises of lower fibers of trapezius muscle, and not because of the traditional physical therapy program. This may be attributed to that the strengthening of the lower fibers of trapezius might have improved the posterior tipping [13]. General functions of the trapezius include scapular upward rotation and elevation for the upper trapezius, retraction for the middle trapezius, and upward rotation and depression for the lower trapezius. In addition, the inferomedial-directed fibers of the lower trapezius may also contribute to the posterior tilt and external rotation of the scapula during arm elevation, thus decreasing subacromial impingement risk and making the lower trapezius an important area to focus on in rehabilitation [29]. The increased lower trapezius muscle activity played an antagonistic role to counteract the increased upper trapezius muscle activity with regard to excessive scapular elevation. The increase in the lower trapezius muscle activation contribute to posterior tipping of the scapula to avoid closer proximity between the anterior acromion and rotator cuff tendons during elevation of the arm [13].

These results may be due to the nature of the treatment program (ultrasound, mobilization and wand exercises), which worked on the limited range of motion rather than strengthening the weak scapulothoracic muscles and correcting the abnormal kinematics. Thus, this may be the reason behind the insignificant difference in scapular tipping in the group that received traditional physical therapy program.

The findings of the current study agreed with Kluemper et al. who compared a $6 \mathrm{w}$ training program that included stretching the anterior shoulder soft tissue and strengthening the posterior shoulder muscles (including strengthening exercises for lower trapezius fibers) with a control group which participated in normal swim-training activities [30]. They concluded that this training routine is the appropriate intervention for correcting forward positional (anterior scapular tipping) or postural fault caused by muscle imbalance. Anterior scapular tipping was measured with a double square method. The subject stood against a wall and the distance between the anterior acromion and the wall was measured. Measurements were taken in two positions, resting and an "upright military" posture before and after a 6 time interval.

In the same context, Celik et al. [31] investigated the effect of adding scapulothoracic strengthening exercises (serratus anterior, middle and lower trapezius, latissimus dorsi) to a physical therapy program in patients with adhesive capsulitis. They concluded that adding scapulothoracic exercises to glenohumeral ROM exercises contributed to the reduction of pain and increased ROM in patients with frozen shoulder. The authors supposed that the group treated with scapular strengthening improved because the scapulohumeral rhythm was restored. However, the outcome variables (functional, pain, and ROM) were different than the current study outcomes.
The results of the current study are also supported by the findings reported by $\mathrm{Hsu}$ et al. [32]. The study proved that taping over the lower fibers of trapezius tended to facilitate the lower fibers of trapezius, consequently increasing the strength of the muscle. This increased the scapular posterior tilt in subjects with shoulder impingement compared to sham taped group.

Recently, Turgut et al. [33] investigated the effects of two different exercise programs on three-dimensional scapular kinematics, in participants with subacromial impingement syndrome. The intervention group was treated with shoulder girdle stretching and strengthening with additional scapular stabilization exercises based on a kinetic chain approach. The control group was treated with shoulder girdle stretching and strengthening exercises only. They noticed an increase in the scapular posterior tilt in both groups. However there was a significant increase in scapular posterior tilting in the intervention group compared with controls [33].

There are some limitations in this study. The first might be the small number of cases recruited. Secondly, there was a lack of follow-up for patients in group B for several months' post rehabilitation program, and this hindered the evaluation of the long lasting effect of the additional effect of strengthening lower fibres of trapezius. In addition, certain secondary outcome variables such as pain, functional activity, and shoulder range of motion were not assessed. In conclusion, this study shows that additional strengthening of lower fibres of trapezius to traditional physical therapy program yields an improvement in scapular tipping in patients with DFS more than traditional physical therapy alone.

\section{References}

1. Page P, Labbe A. Adhesive capsulitis: use the evidence to integrate your interventions. N Am J Sports Phys Ther 2010; 5: 266-273.

2. Kelley M, Shaffer M, Kuhn J, Michener L, Seitz A, Uhl T. Shoulder pain and mobility deficits: adhesive capsulitis. J Orthop Sports Phys Ther 2013; 43: 1-31.

3. Donatelli R, Ruivo R, Thurner M, Ibrahim M. New concepts in restoring shoulder elevation in a stiff and painful shoulder patient. Phys Ther Sport 2014; 15: 3-14.

4. Contractor ES, Agnihotri DS, Patel RM. Effect of spencer muscle energy technique on pain and functional disability in cases of adhesive capsulitis of shoulder joint. IAIM 2016; 3: 126-131.

5. Wang K, Ho V, Hunter-Smith D, Beh P, Smith K, Weber A. Risk factors in idiopathic adhesive capsulitis: a case control study. J Shoulder Elbow Surg 2013; 22: 24-29.

6. Zuckerman JD, Rokito A. Frozen shoulder: a consensus definition. J Shoulder Elbow Surg 2011; 20: 322-325.

7. Neviaser AS, Hannafin JA. Adhesive capsulitis: a review of current treatment. Am J Sports Med 2010; 38: 2346-2356.

8. Hannafin JA, Chiaia TA. Adhesive capsulitis. A treatment approach. Clin Orthop Relat Res 2000; 95-109. 
9. Paine R, Voight ML. The role of the scapula. Int J Sports Phys Ther 2013; 8: 617-629.

10. Kibler W, Ludewig P, McClure P, Michener L, Bak K, Sciascia A. Clinical implications of scapular dyskinesis in shoulder injury: the 2013 consensus statement from the scapular summit. Br J Sports Med 2013; 47: 877-885.

11. Kamkar A, Irrgang J, Whitney S. Nonoperative Management of Secondary Shoulder Impingement Syndrome. J Orthop Sports Phys Ther 1993; 17: 212-224.

12. Jobe F, Pink M. Operative techniques in upper extremity sports injuries. St. Louis Mosby 1996.

13. Lin J, Wu Y, Wang S, Chen S. Trapezius muscle imbalance in individuals suffering from frozen shoulder syndrome. Clin Rheumatol 2005; 24: 569-575.

14. Ludewig PM, Cook TM. Alterations in shoulder kinematics and associated muscle activity in people with symptoms of shoulder impingement. Phys Ther 2000; 80: 276-291.

15. Ludewig P, Reynolds J. The association of scapular kinematics and glenohumeral joint pathologies. J Orthop Sports Phys Ther 2009; 39: 90-104.

16. Suresh K. An overview of randomization techniques: An unbiased assessment of outcome in clinical research. J Human Reprod Sci 2011; 4: 8.

17. World Medical Association. WMA Declaration of Helsinkiethical principles for medical research involving human subjects 2015 .

18. Ibrahim M, Donatelli R, Hellman M, Echternach J. Efficacy of a static progressive stretch device as an adjunct to physical therapy in treating adhesive capsulitis of the shoulder: A prospective, randomised study. Physiotherapy 2014; 100: 228-234.

19. Chen CY, Hu CC, Weng PW. Extracorporeal shockwave therapy improves short-term functional outcomes of shoulder adhesive capsulitis. J Shoulder Elbow Surg 2014; 23: 1843-1851.

20. Host HH. Scapular taping in the treatment of anterior shoulder impingement. PhysTher 1995; 75: 803-812.

21. Nijs J, Roussel N, Vermeulen K, Souvereyns G. Scapular positioning in patients with shoulder pain: a study examining the reliability and clinical importance of 3 clinical tests. Arch Phys Med Rehab 2005; 86: 1349-1355.

22. Struyf F, Nijs J, Mottram S, Roussel NA, Cools AMJ, Meeusen R. Clinical assessment of the scapula: a review of the literature. Br J Sports Med 2014; $48: 883$.
23. Rawat P, Eapen C, Seema K. Effect of rotator cuff strengthening as an adjunct to standard care in subjects with adhesive capsulitis: A randomized controlled trial. J Hand Ther 2017; 30: 235-241.

24. Dogru H, Basaran S, Sarpel T. Effectiveness of therapeutic ultrasound in adhesive capsulitis. Joint Bone Spine 2008; 75: 445-450.

25. Kisner C, Colby L, Borstad J. Therapeutic exercise: foundations and techniques. FA Davis 2012.

26. Arlotta M, Lovasco G, McLean L. Selective recruitment of the lower fibers of the trapezius muscle. J Electromyogr Kinesiol 2011; 21: 403-410.

27. Ekstrom R, Donatelli R, Soderberg G. Surface electromyographic analysis of exercises for the trapezius and serratus anterior muscles. J Orthop Sports Phys Ther 2003; 33: 247-258.

28. Chaitow L, Franke H, Chaitow L. Muscle energy techniques. Edinburgh: Churchill Livingstone/Elsevier 2013.

29. Ludewig PM, Cook TM. Alterations in shoulder kinematics and associated muscle activity in people with symptoms of shoulder impingement. Phys Ther 2000 ; 80: 276-291.

30. Kluemper M, Uhl T, Hazelrigg H. Effect of stretching and strengthening shoulder muscles on forward shoulder posture in competitive swimmers. J Sport Rehab 2006; 15: 58-70.

31. Celik D. Comparison of the outcomes of two different exercise programs on frozen shoulder. Acta Orthopaedica et Traumatologica Turcica 2010; 44: 285-292.

32. Hsu JE, Anakwenze OA, Warrender WJ, Abboud JA. Current review of adhesive capsulitis. J Shoulder Elbow Surg 2011; 20: 502-514.

33. Turgut E, Duzgun I, Baltaci G. Effects of scapular stabilization exercise training on scapular kinematics, disability, and pain in subacromial impingement: a randomized controlled trial. Arch Phys Med Rehab 2017.

\section{*Correspondence to}

Hamada Hamada

Lecture of Biomechanics

Faculty of Physical Therapy

Cairo University 